\title{
Rank Minimization and Applications in System Theory
}

\author{
M. Fazel, H. Hindi, and S. Boyd
}

\begin{abstract}
In this tutorial paper, we consider the problem of minimizing the rank of a matrix over a convex set. The Rank Minimization Problem (RMP) arises in diverse areas such as control, system identification, statistics and signal processing, and is known to be computationally NP-hard. We give an overview of the problem, its interpretations, applications, and solution methods. In particular, we focus on how convex optimization can be used to develop heuristic methods for this problem.
\end{abstract}

\section{INTRODUCTION}

In many engineering äpplications, notions such as order, complexity, or dimension of a model or design can be expressed as the rank of a matrix. If the set of feasible models or designs is described by convex constraints, then choosing the simplest model can often be expressed as a Rank Minimization Problem (RMP). For example, a lowrank matrix could correspond to a low-order controller for a system, a low-order statistical model fit for a random process, a shape that can be embedded in a low-dimensional space, or a design with a small number of components. It is not surprising that rank minimization has such a wide range of applications across all disciplines of engineering and computational sciences: we are often interested in simple models. This idea is well captured by the principle known as Occam's razor, which states that "Among competing explanations for a phenomenon, the simplest one is the best."

There are several special cases of the RMP that have well known solutions. For example, approximating a given matrix with a low-rank matrix in spectral or Frobenius norm is an RMP that can be solved via singular value decomposition (SVD) [15]. However, in general, the RMP is known to be computationally intractable (NP-hard) [26].. Therefore, we do not expect to find a computationally efficient (polynomial-time) method that can solve all instances of the problem exactly. And unless the number of variables in the problem is very small, global search methods, that have exponential time complexity, are not viable options. Therefore, what we discuss here are heuristics that solve the problem approximately but efficiently.

The structure of the paper is as follows. In sections II and III we define the RMP and demonstrate its meaning in different contexts and applications. In section IV, we give an overview of various heuristic solution methods.

M. Fazel is with the Control and Dynamical Systems Dept, Caltech, Pasadena, CA. maryam@cds. caltech. edu

H. Hindi is with the Palo Alto Research Center (PARC), Palo Alto, CA. Haitham.Hindi@parc.com

S. Boyd is with the Information Systems Lab, Stanford University, Stanford, CA. boyd@stanford.edu

Research conducted while all authors were at Stanford University.
In particular, we focus on recently developed heuristics based on convex optimization, i.e., the trace and log-det heuristics [7], [8]. Section V demonstrates the use of the heuristics for the problem of system realization with time domain constraints.

\section{The Rank Minimization Problem}

The general RMP can be expressed as

$$
\text { RMP: minimize } \operatorname{Rank} X
$$

where $X \in \mathbf{R}^{m \times n}$ is the optimization variable and $\mathcal{C}$ is a convex set denoting the constraints.

As a generic example of the RMP, suppose we are trying to estimate or reconstruct the covariance matrix

$$
X=\mathbf{E}(z-\mathbf{E} z)(z-\mathbf{E} z)^{T}
$$

of a random vector $z \in \mathbf{R}^{n}$, from measurements and prior assumptions. Here $\mathbf{E}(z)$ denotes the expectation of the random vector $z$. The constraint $X \in \mathcal{C}$ expresses the condition that the estimated covariance matrix is consistent with (or not improbable for) our measurements or observed data and prior assumptions. For example, it could mean that entries in $X$ should lie in certain intervals. The rank of $X$ is a measure of the complexity of the stochastic model of $z$, in the sense that it gives the number of underlying independent random variables needed to explain the covariance of $z$. The RMP (1) is therefore the problem of finding the least complex stochastic model (i.e., covariance) that is consistent with the observations and prior assumptions. As we will point out in the next section, this problem has many practical applications. In other applications, rank can have other meanings such as embedding dimension, controller order, or number of signals present.

In problem (1), we allow any constraints on the matrix as long as they describe a convex set. Thus, we cover a large number of constraints and specifications that come up in practice. For example, constraints on the accuracy of a model or the performance of a design are common; e.g., $f(X) \leq t$, where $f(\cdot)$ is a (convex) measure of performance, and $t \in \mathbf{R}$ is the tolerance. We give examples of these constraints in the next section.

\section{APPLications OF THE RMP}

\section{A. Rank of a covariance matrix}

Problems involving the rank of a covariance matrix often arise in statistics, econometrics, signal processing, and other fields where second-order statistics for random processes are used. Second-order statistical data analysis methods, such as principal component analysis and factor analysis, 
deal with covariance matrices estimated from noisy data. Because of noise, the estimated covariance matrices have full rank (with probability one). Finding a covariance matrix of low rank comes up naturally in these methods. A lowrank covariance matrix corresponds to a simple explanation or model for the data. For example, consider the following constrained factor analysis problem:

$$
\begin{array}{ll}
\operatorname{minimize} & \operatorname{Rank}(\Sigma) \\
\text { subject to } & \|\Sigma-\hat{\Sigma}\|_{F} \leq \epsilon, \\
& \Sigma \geq 0 \\
& \Sigma \in \mathcal{C},
\end{array}
$$

where $\Sigma \in \mathbf{R}^{n \times n}$ is the optimization variable, $\hat{\Sigma}$ is the measured covariance matrix, $\mathcal{C}$ is a convex set denoting the prior information or assumptions on $\Sigma$, and $\|\cdot\|_{F}$ denotes the Frobenius norm of a matrix (other matrix norms can be handled as well). The constraint $\|\Sigma-\hat{\Sigma}\|_{F} \leq \epsilon$ means that the error, i.e., the difference between $\Sigma$ and the measured covariance in Frobenius norm, must be less than a given tolerance $\epsilon$. The constraint $\Sigma \geq 0$ ensures that we obtain a valid covariance matrix. In the statistics terminology, the objective function, $\operatorname{Rank} \Sigma$ corresponds to the number of factors that explain $\Sigma$.

If $\mathcal{C}=\mathbf{R}^{n \times n}$ (i.e., no prior information), this problem has an SVD-based analytical solution. However, extra constraints such as upper and lower bounds on the entries of $\Sigma$ result in a computationally hard problem.

\section{B. Rank of a Hankel matrix}

We saw in the previous section that the rank of a covariance matrix plays a central role in many statistical methods as a notion of complexity of the stochastic model. The rank of a Hankel matrix has similar significance in model identification problems in system theory and signal processing. It comes up commonly in problems that deal with recursive sequences, where the order of the recursion is expressed by the rank of an appropriate Hankel matrix.

Problems involving minimizing the rank of a Hankel matrix also come up in system realization, e.g., in designing a low-order linear, time-invariant system directly from convex specifications on its impulse response. We discuss this problem is section $\mathrm{V}$.

\section{Other examples}

RMPs have been studied extensively in the control literature, since many important problems in controller design and system identification can be expressed as an RMP. Minimum-order controller design is perhaps the most widely studied problem among these (sce, e.g.,[9], [17]). Another problem is model order reduction in system identification. Other applications include reduced-order $\mathcal{H}_{\infty}$ synthesis and reduced-order $\mu$ synthesis with constant scalings [4], problems with inertia constraints [13], exact reducibility of uncertain systems [1], and simultaneous stabilization of linear systems [14].

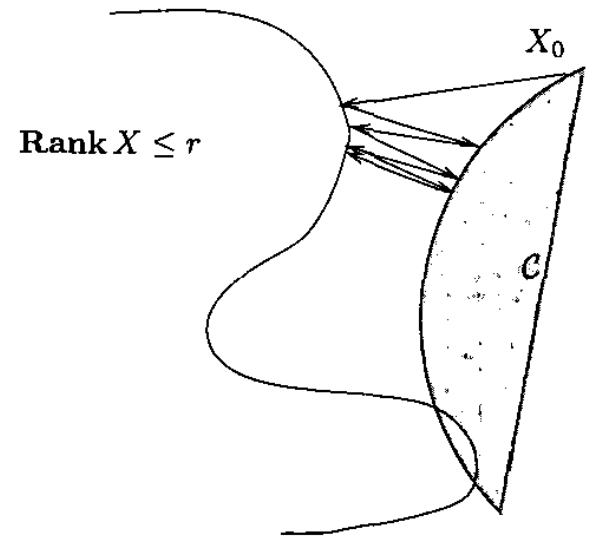

Fig. 1: Illustration of the alternating projections method for the RMP.

Low-rank matrix approximations are also sometimes used to save computational effort. As a simple example, suppose we want to compute $y=A x$, where $A \in \mathbf{R}^{m \times n}$, for various values of $x$, and suppose $m$ and $n$ are large. This requires $m n$ multiplications. If $\operatorname{Rank} A=r$, then $A$ can be factored as $A=R L^{T}$, where $R \in \mathbf{R}^{m \times r}$ and $L \in \mathbf{R}^{m \times r}$. Thus, $y=R L^{T} x$ can be computed with only $(m+n) r$ multiplications. If $r$ is much smaller than $m$ and $n$, this could lead to significant savings in computation. The simplest matrix approximation problem is

$$
\begin{array}{ll}
\text { minimize } & \operatorname{Rank} \hat{A} \\
\text { subject to } & \|A-\hat{A}\| \leq \epsilon,
\end{array}
$$

where $\hat{A}$ is the optimization variable and $\epsilon$ is the tolerance. This problem can readily be solved via SVD. However, often when $A$ has a particular structure (e.g., Hankel or Toeplitz), $\hat{A}$ is desired to retain that structure. Such additional constraints typically make the problem hard.

\section{SOLUTION APPROACHES}

In this section we discuss solution approaches to the RMP. We list some existing approaches organized into three groups, and review them briefly. We then focus in detail on two heuristics based on convex optimization, i.e., the trace and log-det heuristics.

\section{A. Alternating projections method}

This method is based on the fact that the sequence of orthogonal projections onto two closed, convex sets converges to a point in the intersection of the sets, if the intersection is non-empty [11]. If one or more of the sets are non-convex, convergence to the intersection is no longer guaranteed. In this case, we can have a situation where the sets intersect but the sequence of projections converges to a limit cycle, as depicted in figure 1 . However, local convergence is still guaranteed and the method may be used as a heuristic. The alternating projections method can be applied to the RMP (this is used in [2] for the low-order controller design problem). Wc first fix the desired rank $r$. The goal is to 
find a matrix in the intersection of the following two sets, or determine that the intersection is empty: (i) the set of matrices of rank $r$, and (ii) the constraint set $\mathcal{C}$. Note that the first set is nonconvex. Projection onto the set of matrices of rank $r$, i.e., finding the closest rank $r$ matrix to the current iterate $X_{k-1}$, can be done by SVD. We denote the solution by $\tilde{X}_{k}$. Projection onto the constraint set $\mathcal{C}$ can be done by minimizing the distance from $\tilde{X}_{k}$ to the set $\mathcal{C}$. In summary, given a desired value of rank $r$, we use the following algorithm to check whether there is any $X \in \mathcal{C}$ such that $\operatorname{Rank} X \leq r$ :

- Choose $X_{0} \in \mathcal{C}$. Set $k=1$.

- repeat

$$
\begin{aligned}
& \tilde{X}_{k}=\sum_{i=1}^{r} \lambda_{i} u_{i} v_{i}^{T}, \quad \text { where } X_{k-1}=U \Sigma V^{T}, \\
& X_{k}=\operatorname{argmin}_{X \in \mathcal{C}}\left\|X-\tilde{X}_{k}\right\|, \\
& e_{k}=\left\|X_{k}-\tilde{X}_{k}\right\|, \\
& \text { until }\left|e_{k}-e_{k-1}\right| \leq \epsilon
\end{aligned}
$$

See [10, chapter 10$]$ and references therein for a detailed discussion of the alternating projection method and its variations, and their application to low-order control design.

In general, this method is known to have slow convergence [24], although there have been recent variations that improve the speed [24, chapter 10]. Also note that in each iteration, in addition to an SVD, we need to solve the problem of projection onto $\mathcal{C}$. In some special cases, this projection has a simple analytical expression (see [24]). In these cases, we can afford a large number of iterations since the computation required per iteration is very low; but in general, each iteration involves solving a convex problem, e.g., a semidefinite program.

B. Factorization, coordinate descent and linearization methods

The idea behind factorization methods is that $\operatorname{Rank}(X) \leq r$ if and only if $X$ can be factored as $X=F G^{T}$, where $F \in \mathbf{R}^{m \times r}$ and $G \in \mathbf{R}^{n \times r}$. For each given $r$, we check if there exists a feasible $X$ of rank less than or equal to $r$ by checking if any $X \in \mathcal{C}$ can be factored as above.

The expression $X=F G^{T}$ is not convex in $X, F$, and $G$ simultaneously, but it is convex in $(X, F)$ when $G$ is fixed, and convex in $(X, G)$ when $F$ is fixed. Various heuristics can be applied to handle this non-convex equality constraint. We consider the following simple heuristic: Fix $F$ and $G$ one at a time and iteratively solve a convex problem at each step. This can be expressed as

- Choose $F_{0} \in \mathbf{R}^{m \times r}$. Set $k=1$.

- repeat

$$
\begin{aligned}
& \left(\tilde{X}_{k}, G_{k}\right)=\underset{X \in \mathcal{C}, G \in \mathbf{R}^{n \times r}}{\operatorname{argmin}}\left\|X-F_{k-1} G^{T}\right\|_{F} \\
& \left(X_{k}, F_{k}\right)=\underset{X \in \mathcal{C}, F \in \mathbf{R}^{m \times r}}{\operatorname{argmin}}\left\|X-F G_{k}^{T}\right\|_{F} \\
& e_{k}=\left\|X_{k}-F_{k} G_{k}^{T}\right\|_{F}, \\
& \text { until } e_{k} \leq \epsilon \text {, or } e_{k} \text { and } e_{k-1} \text { are approximately equal. }
\end{aligned}
$$

This is a coordinate descent method, since some variables (i.e., coordinates) are fixed during each minimization step.

Another heuristic to handle the non-convex constraint $X=F G^{T}$ is to linearize this equation in $F$ and $G$. Assuming the perturbations $\delta F, \delta G$ are small enough so that the second order term is negligible, we get $X=$ $F G^{T}+F \delta G^{T}+\delta F G^{T}$. This constraint can be handled easily since it is linear in both $\delta F$ and $\delta G$. The method is useful if the initial choice for $F G^{T}$ is close enough to a rank $r$ matrix for the small perturbations assumption to be valid. This method has been used in BMI problems that come up in low-authority controller design [12].

Some other heuristics, similar to the ones described here, have been applied to the problem of reduced order controller design in the control literature. This problem has a particular structure, allowing for different choices for the variables in a coordinate descent or linearization method. For example, the dual iteration method in [16] and the successive minimization approach in [24] are coordinate descent methods applied to this problem, and [5] gives a linearization method based on a cone-complementarity formulation.

\section{Interior-point-based methods}

Consider a positive semidefinite RMP, i.e., a special case of the RMP with the extra constraint that $X \geq 0$. Reference [3] proposes heuristics for this problem that use ideas from interior point methods for convex optimization [20]. One heuristic, called analytic anti-centering, is based on the properties of convex logarithmic barrier functions; specifically, that they grow to infinity as the boundary of the feasible set is approached. Minimization of a log-barrier function using the Newton method produces a point in the interior of the feasible set, known as the analytic center. Now note that any rank-deficient solution to the positive semidefinite RMP must lie on the boundary of the semidefinite cone. The analytic anti-centering approach takes steps in the reverse Newton direction, in order to maximize the log-barrier function. This tends to produce points that are on the boundary of the feasible set, and hence rank deficient. Since this approach involves the maximization of a convex function, the solutions are not necessarily global optima.

Note that in these methods the result is highly sensitive to the choice of the initial point. The initial point is typically chosen in the vicinity of the analytic center of the feasible region. The iterations may follow a completely different path to a different point on the boundary if the initial point is slightly changed. See reference [3] for more details and examples, and for the application of these methods to loworder control design.

In the next section, we focus our attention on heuristics based on solving convex problems, and present a few useful properties of these methods. 


\section{Trace and Log-det heuristics}

A well-known heuristic for the RMP when the variable $X \in \mathbf{R}^{n \times n}$ is positive semidefinite is to replace the rank objective in (1) with the trace of $X$ and solve

$$
\begin{array}{ll}
\text { minimize } & \operatorname{Tr} X \\
\text { subject to } & X \in \mathcal{C} \\
& X \geq 0 .
\end{array}
$$

One way to see why this heuristic works is to note that $\operatorname{Tr} X=\sum_{i=1}^{n} \lambda_{i}(X)$, where $\lambda_{i}(X)$ are the eigenvalues of $X$. This is the same as $\|\lambda(X)\|_{1}=\sum_{i=1}^{n}\left|\lambda_{i}(X)\right|$ for a PSD matrix where the eigenvalues are non-negative. It is known that to obtain a sparse vector, minimizing the $\ell_{1}$-norm of the vector is an effective heuristic [18], [8]. Thus, minimizing the $\ell_{1}$-norm of $\lambda(X)$ renders many of the eigenvalues as zero, resulting in a low-rank matrix. The trace heuristic has been used in many applications; see for example [22], [23]. Also see [19] for a special case where this heuristic is exact. Trace heuristic's popularity stems from the fact that problem (3) is a convex optimization problem, which can be solved very efficiently and reliably in practice.

The log-det heuristic can be described as follows: rather than solving the RMP, use the function $\log \operatorname{det}(X+\delta I)$ as a smooth surrogate for $\operatorname{Rank} X$ and instead solve the problem

$$
\begin{array}{ll}
\text { minimize } & \log \operatorname{det}(X+\delta I) \\
\text { subject to } & X \in \mathcal{C},
\end{array}
$$

where $\delta>0$ can be interpreted as a small regularization constant. Note that the surrogate function $\log \operatorname{det}(X+\delta I)$ is not convex (in fact, it is concave). However, since it is smooth on the positive definite cone, it can be minimized (locally) using any local minimization method. We use iterative linearization to find a local minimum. Let $X_{k}$ denote the $k$ th iterate of the optimization variable $X$. The first-order Taylor series expansion of $\log \operatorname{det}(X+\delta I)$ about $X_{k}$ is given by

$$
\begin{aligned}
& \log \operatorname{det}(X+\delta I) \approx \\
& \quad \log \operatorname{det}\left(X_{k}+\delta I\right)+\operatorname{Tr}\left(X_{k}+\delta I\right)^{-1}\left(X-X_{k}\right) .
\end{aligned}
$$

Here we have used the fact that $\nabla \log \operatorname{det} X=X^{-1}$, when $X>0$. Hence, one could attempt to minimize $\log \operatorname{det}(X+$ $\delta I$ ) over the constraint set $\mathcal{C}$ by iteratively minimizing the local linearization (5). This leads to

$$
X_{k+1}=\underset{X \in \mathcal{C}}{\operatorname{argmin}} \operatorname{Tr}\left(X_{k}+\delta I\right)^{-1} X .
$$

The new optimal point is $X_{k+1}$, and we have ignored the constants in (5) because they do not affect the minimization. Since the function $\log \operatorname{det}(X+\delta I)$ is concave in $X$, at each iteration its value decreases by an amount more than the decrease in the value of the linearized objective. Based on this observation, it can be shown that the sequence of the function values generated converges to a local minimum of $\log \operatorname{det}(X+\delta I)$.

Note that the trace heuristic can be viewed as the first iteration in (6), starting from the initial point $X_{0}=I$.
Therefore, we always pick $X_{0}=I$, so that $X_{1}$ is the result of the trace heuristic, and the iterations that follow try to reduce the rank of $X_{1}$ further.

1) Generalized Trace and Log-det heuristics: The two heuristics given in the previous section are applicable directly only to RMPs where the matrix variable is positive semidefinite. However, using the following embedding lemma both heuristics are readily extended to handle general matrices.

Lemma 1: Let $X \in \mathbf{R}^{m \times n}$ be a given matrix. Then $\operatorname{Rank} X \leq r$ if and only if there exist matrices $Y=Y^{T} \in$ $\mathbf{R}^{m \times m}$ and $Z=Z^{T} \in \mathbf{R}^{n \times n}$ such that

$$
\operatorname{Rank} Y+\operatorname{Rank} Z \leq 2 r, \quad\left[\begin{array}{cc}
Y & X \\
X^{T} & Z
\end{array}\right] \geq 0 .
$$

The proof is omitted here, in the interest of space and the tutorial nature of this paper. For the proof, see [8].

This result means that minimizing the rank of a general nonsquare matrix $X$, problem (1), is equivalent to minimizing the rank of the semidefinite, block diagonal matrix $\operatorname{diag}(Y, Z)$ :

$$
\begin{array}{ll}
\operatorname{minimize} & \frac{1}{2} \operatorname{Rank} \operatorname{diag}(Y, Z) \\
\text { subject to } & {\left[\begin{array}{cc}
Y & X \\
X^{T} & Z
\end{array}\right] \geq 0} \\
& X \in \mathcal{C},
\end{array}
$$

with variables $X, Y$ and $Z$ (we drop the constant factor $1 / 2$ from now on).

Using this lemma, to have a generalized version of the trace heuristic we simply apply the trace heuristic to (8) by replacing Rank with Tr. The resulting heuristic can be shown [7] to be equivalent to the following problem,

$$
\begin{array}{ll}
\text { minimize } & \|X\|_{*} \\
\text { subject to } & X \in \mathcal{C},
\end{array}
$$

where $\|X\|_{*}=\sum_{i=1}^{\min \{m, n\}} \sigma_{i}(X)$ is called the nuclear norm or the $K y$-Fan n-norm of $X$; see, e.g., [15]. Here $\sigma_{i}(X)$ are the singular values of $X$. This norm is the dual of the spectral (or the maximum singular value) norm. The following theorem yields an interesting interpretation of the nuclear norm heuristic: in effect, this heuristic minimizes the convex envelope of the rank function over a bounded set (see [6] for the proof and more details).

Theorem 1: On the set $\mathcal{S}=\left\{X \in \mathbf{R}^{m \times n} \mid\|X\| \leq 1\right\}$, the convex envelope of the function $\phi(X)=\operatorname{Rank} X$ is $\phi_{\mathrm{env}}(X)=\|X\|_{*}=\sum_{i=1}^{\min \{m, n\}} \sigma_{i}(X)$.

The convex envelope of $f: \mathcal{C} \rightarrow \mathbf{R}$ is defined as the largest convex function $g$ such that $g(x) \leq f(x)$ for all $x \in \mathcal{C}$ (Figure 2). This means that among all convex functions, $g$ is the one that is closest (pointwise) to $f$. In situations such as problem (1) where the objective function is non-convex, its convex envelope can serve as a tractable convex approximation that can be minimized efficiently. The minimum of the convex envelope can then serve as a lower bound on the true minimum, and the minimizing argument can serve as an initial point for a 


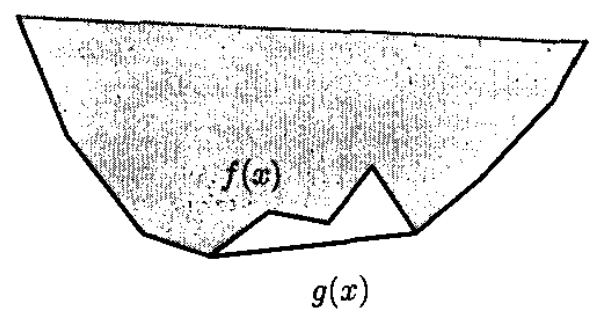

Fig. 2: Illustration of convex envelope of a function.

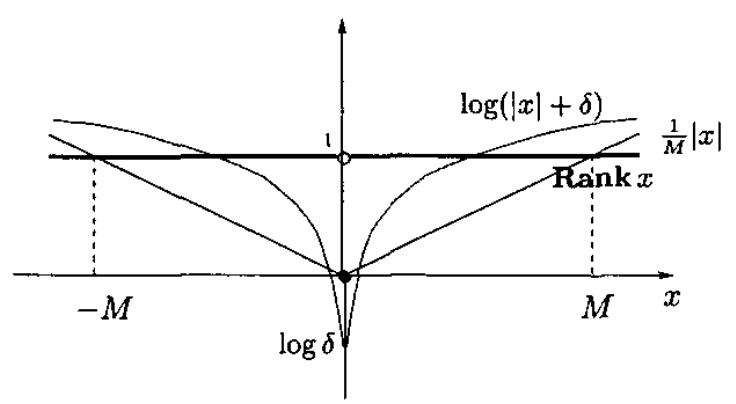

Fig. 3: The rank, trace, and log-det objectives in the scalar case.

more complicated non-convex local search method, e.g., the generalized log-det heuristic that we will discuss next.

In order to extend the log-det heuristic to the general case, we appeal to Lemma 1 again. Since the matrix $\operatorname{diag}(Y, Z)$ is semidefinite, the log-det heuristic (4) can be applied. This yields

$$
\begin{array}{ll}
\text { minimize } & \log \operatorname{det}(\operatorname{diag}(Y, Z)+\delta I) \\
\text { subject to } & {\left[\begin{array}{cc}
Y & X \\
X^{T} & Z
\end{array}\right] \geq 0} \\
& \\
X \in \mathcal{C} . &
\end{array}
$$

Linearizing as before, we obtain the following iterations for solving (10) locally:

$$
\begin{gathered}
\operatorname{diag}\left(Y_{k+1}, Z_{k+1}\right)= \\
\operatorname{argmin} \operatorname{Tr}\left(\operatorname{diag}\left(Y_{k}, Z_{k}\right)+\delta I\right)^{-1} \operatorname{diag}(Y, Z) \\
\text { subject to }\left[\begin{array}{cc}
Y & X \\
X^{T} & Z
\end{array}\right] \geq 0 \\
X \in \mathcal{C},
\end{gathered}
$$

where each iteration is an SDP in the variables $X, Y$ and $Z$.

Figure 3 provides an intuitive interpretation for the heuristic. It shows the basic idea behind the $\operatorname{Tr} X$ and $\log \operatorname{det}(X+\delta I)$ approximations of Rank $X$. The objective functions for the trace and log-det heuristics are shown for the scalar case, i.e., when $x \in \mathbf{R}$ and $\sigma(x)=|x|$.

\section{APPLICATION EXAMPLE: SYSTEM REALIZATION WITH TIME-DOMAIN CONSTRAINTS}

In this section, we discuss the problem of designing a low-order, discrete-time, linear time-invariant (LTI) dynamical system, directly from convex specifications on the first $n$ time samples of its impulse response. Some typical specifications are bounds on the rise-time, settling-time, slew-rate, overshoot, etc. This problem can be posed as one of minimizing the rank of a Hankel matrix over a convex set.

We begin with a fact about linear systems that can be derived from standard results in [25]. We denote by $H_{n}$ the Hankel matrix with parameters $h_{1}, h_{2}, \ldots, h_{2 n-1} \in \mathbf{R}$,

$$
H_{n}=\left[\begin{array}{ccccc}
h_{1} & h_{2} & h_{3} & \ldots & h_{n} \\
h_{2} & h_{3} & h_{4} & \ldots & h_{n+1} \\
h_{3} & h_{4} & h_{5} & \ldots & h_{n+2} \\
\vdots & \vdots & \vdots & \ddots & \vdots \\
h_{n} & h_{n+1} & h_{n+2} & \ldots & h_{2 n-1}
\end{array}\right]
$$

Fact 1: Let $h_{1}, h_{2}, \ldots, h_{n}$ be given real numbers. Then there exists a minimal LTI system of order $r$, with state space matrices $A \in \mathbf{R}^{r \times r}, b \in \mathbf{R}^{r \times 1}$ and $c \in \mathbf{R}^{1 \times r}$, such that

$$
c A^{i-1} b=h_{i} \quad i=1, \ldots, n,
$$

if and only if

$$
r=\min _{h_{n+1}, \ldots, h_{2 n-1} \in \mathbf{R}} \operatorname{Rank} H_{n},
$$

where $H_{n}$ is a Hankel matrix whose first $n$ parameters are the given $h_{1}, h_{2}, \ldots, h_{n}$, and whose last $n-1$ parameters, $h_{n+1}, \ldots, h_{2 n-1} \in \mathbf{R}$, are free variables.

In other words, there exists a linear time invariant system of order $r$ whose first $n$ impulse response samples are $h_{1}, \ldots, h_{n}$, if and only if the minimal-rank Hankel matrix has rank $r$. Once $h_{1}, \ldots, h_{2 n-1}$ are known, a state space description $\{A, b, c\}$ can be easily obtained [21].

Note that the constraints in the Fact 1 are only on the first $n$ samples, even though $h_{n+1}, \ldots, h_{2 n-1}$ also appear in the Hankel matrix. These extra variables are left free in the optimization. Thus, they are chosen in a way so as to minimize the overall rank of the Hankel matrix.

To see how the facts above can be used to design loworder systems directly from specifications, consider the specifications on the step response shown in Figure 4. The goal is to find the minimum-order system whose step response fits in the region defined by the dashed lines, up to the 16th sample. The dashed lines are meant to capture a typical set of time-domain step response specifications: certain rise-time, slew-rate, overshoot, and settling characteristics and an approximate delay of four samples. The problem can be expressed as

$$
\begin{array}{ll}
\text { minimize } & \operatorname{Rank} H_{n} \\
\text { subject to } & l_{i} \leq s_{i} \leq u_{i}, \quad k=1, \ldots, n \\
& h_{n+1}, \ldots, h_{2 n-1} \in \mathbf{R},
\end{array}
$$

where $s_{k}=\sum_{i=1}^{k} h_{i}$ denote the terms in the step response, and $l_{i}$ and $u_{i}$ are, respectively, samples of the lower and upper time domain specifications (shown by the dashed lines). 


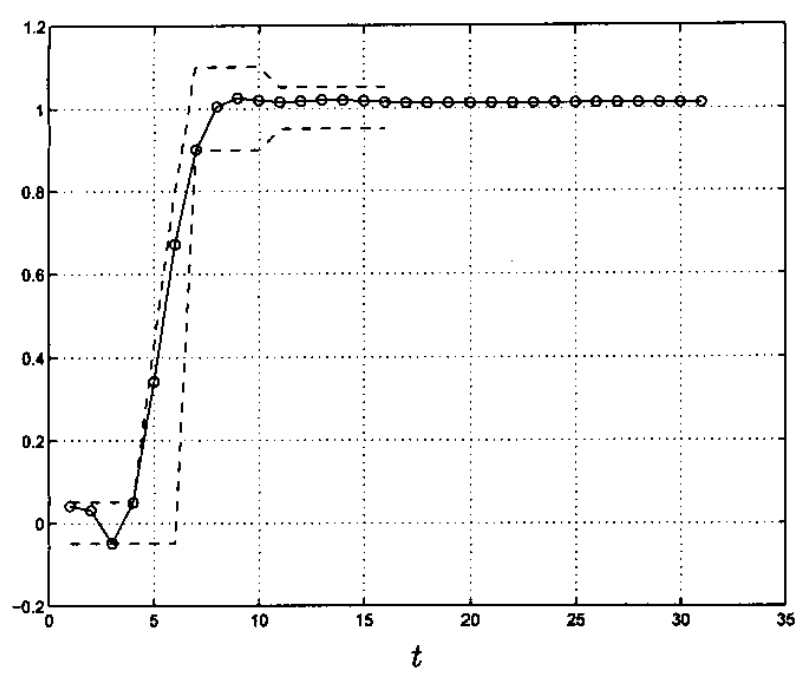

Fig. 4: Step response specs (dashed) and actual step response.

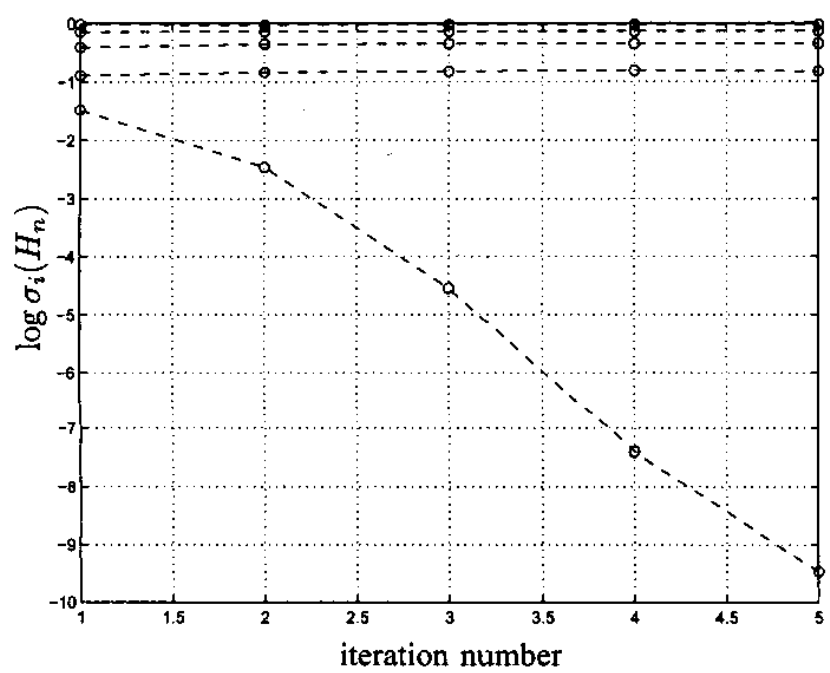

Fig. 5: $\log$ of the singular values of $H_{n}$ at each iteration

This problem is an RMP with no analytical solution. Note also that the optimization variable $H_{n}$ is not positive semidefinite. We apply the generalized trace and log-det heuristics described before to this problem. Because of the approximate four-sample delay specification, we do not expect that the specifications can be met by a system of order less than four.

After five iterations of the log-det heuristic, a fourth-order system is obtained with the step response shown in Figure 4. Thus, all the specifications can be met by a linear timeinvariant system of order exactly four. In this example, we set $\delta=10^{-6}$. Figure 5 shows the logarithm of the nonzero Hankel singular values. We see that the rank of the $16 \times$ 16 matrix $H_{n}$ drops to 5 after the first iteration, and the next four iterations bring the rank to 4 , which in this case happens to be the global minimum.

\section{REFERENCES}

[1] C. Beck and J. Doyle. A necessary and suffi cient minimality condition for uncertain systems. IEEE Trans. Aut. Control, 44(10):1802-1813, October 1999.

[2] E. Beran and K. Grigoriadis. A combined alternating projection and semidefi nite programming algorithm for low-order control design. In Proceedings of IFAC 96, volume C, pages 85-90, July 1996.

[3] J. David. Algorithms for analysis and design of robust controllers. $\mathrm{PhD}$ thesis, Kat. Univ, Leuven, ESAT, 3001 Leuven, Belgium, 1994.

[4] L. El Ghaoui and P. Gahinet. Rank minimization under LMI constraints: A framework for output feedback problems. In Proc. European Control Conf., The Netherlands, 1993.

[5] L. El Ghaoui, F. Oustry, and M. AitRami. A cone complementarity linearization algorithm for static output-feedback and related problems. In Proceedings of the 1996 IEEE International Symposium on ComputerAided Control System Design, pages 246-251, Dearbom, MI, 1996.

[6] M. Fazel Matrix Rank Minimization with Applications. PhD thesis, Stanford University, CA, USA, 2002.

[7] M. Fazel, H. Hindi, and S. P. Boyd. A rank minimization heuristic with application to minimum order system approximation. In Proc. American Control Conf., Arlington, Virginia, 2001.

[8] M. Fazel, H. Hindi, and S. P. Boyd. Log-det heuristic for matrix rank minimization with applications to Hankel and Euclidean distance matrices In Proc. American Control Conf., Denver, Colorado, 2003.

[9] P. Gahinet and P. Apkarian. A linear matrix inequality approach to $\mathcal{H}_{\infty}$ control. Int. J. Robust and Nonlinear Control, 4:421-488, 1994.

[10] K. M. Grigoriadis and E. B. Beran. Altemating projection algorithms for linear matrix inequalities problems with rank constraints. In L. El Ghaoui and S. Niculescu, editors, Advances in Linear Matrix Inequality Methods in Control, chapter 13, pages 251-267. SIAM, 2000.

[11] L. G. Gubin, B. T. Polyak, and E. V. Raik. The method of projections for fi nding the common point of convex sets. USSR Comp. Math. Phys. (7): 1-24, 1967.

[12] A. Hassibi, J. P. How, and S. P. Boyd. A path-following method for solving BMI problems in control. In Proceedings of American Control Conference, volume 2, pages 1385-9, June 1999.

[13] A. Helmersson. IQC synthesis based on inertia constraints. In Proceedings of the 14th IFAC conference, pages 163-168, 1999.

[14] D. Henrion, S. Tarbouriech, and M. Sebek. Rank-one LMI approach to simultaneous stabilization of linear systems. Syst. Control Letters, 38(2):79-89, 1999.

[15] R. Hom and C. Johnson. Topics in Matrix Analysis. Cambridge University Press, Cambridge, 1991.

[16] T. Iwasaki. The dual iteration for fi xed-order control. IEEE Trans. Aut. Control, 44(4):783-788, 1999.

[17] T. Iwasaki and R. E. Skelton. All controllers for the general $\mathbf{H}_{\infty}$ control problem: LMI existence conditions and state space formulas. Automatica, 30(8):1307-1317, 1994.

[18] M. S. Lobo, M. Fazel, and S. Boyd. Portfolio optimization with linear and fixed transaction costs and bounds on risk. Available at www. stanford.edu/ boyd

[19] M. Mesbahi and G. P. Papavassilopoulos. On the rank minimization problem over a positive semidefi nite linear matrix inequality. IEEE Transactions on Automatic Control, AC-42(2):239-43, February 1997.

20] Y. Nesterov and A. Nemirovskii. Interior-Point Polynomial Algorithms in Convex Programming. SIAM Studies in Applied Mathematics. SIAM, 1994.

[21] P. Van Overschee and B. De Moor. Subspace Identification for Linear Systems: Theory, Implementation, Applications. Kluwer, 1996.

[22] T. E. Pare. Analysis and control of nonlinear systems. PhD thesis, Dept. of Aeronautics and Astronautics, Stanford University, August 2000.

[23] P. A. Parrilo. Structured Semidefinite Programs and Semialgebraic Geometry Methods in Robustness and Optimization. PhD thesis, California Institute of Technology, Pasadena, California, 2000.

[24] R. E. Skelton, T. Iwasaki, and K. Grigoriadis. A Unified Algebraic Approach to Linear Control Design. Taylor and Francis, 1998.

[25] E. D. Sontag. Mathematical Control Theory, volume 6 of Texts in Applied Mathematics. Springer-Verlag, 1990.

[26] L. Vandenberghe and S. Boyd. Semidefi nite programming. SIAM Review, 38(1):49-95, March 1996. 